\title{
AOR
}

Selected Papers of \#AolR2020:

The 22nd Annual Conference of the Association of Internet Researchers Virtual Event / 13-16 Oct 2021

\section{RECASTING COLLEGIATE ESPORTS: INDEPENDENCE AND INTERDEPENDENCE OF UNIVERSITY TWITCH STREAMERS}

\author{
Maxwell Foxman \\ University of Oregon \\ Amanda Cote \\ University of Oregon \\ Onder Can \\ University of Oregon \\ Brandon Harris \\ University of Oregon \\ Waseq Rahman \\ University of Oregon \\ Jared Hansen \\ University of Oregon \\ Tara Fickle \\ University of Oregon

\section{Introduction}

Few online communities exhibit tensions between independence and interdependence better than collegiate esports. Competitive gaming on US campuses depends on investments from innumerable stakeholders, including universities, game publishers, and distribution platforms. These interest groups cooperate towards esports' overall success, but each also brings its own interests to the table. The result is often conflicted: from struggles to adhere to Title IX policies to reconsideration of how undergraduate athletes are paid (e.g., Baker \& Holden, 2018). The live-streaming platform Twitch is central in such strife. Twitch is a vital tool in contemporary digital media literacy (Gerber, 2017), widely utilized by esports players (Li et al., 2020), and fosters communities of practice (Richard et al., 2018). It is also the predominant means

Foxman, M., Cote, A., Can, O., Harris, B., Rahman, W., Hansen, J., and Fickle, T. (2021, October). Recasting Collegiate Esports: Independence and Interdependence of University Twitch Streamers. Paper presented at AolR 2021: The 22nd Annual Conference of the Association of Internet Researchers. Virtual Event: AolR. Retrieved from http://spir.aoir.org. 
of esports spectatorship (e.g., Richard et al., 2018; Taylor, 2020); and its streams produce valuable data against which collegiate players measure and analyze their performances (Taylor, 2020). A pathway for entrepreneurship (Anderson et al., 2018), Twitch is the bedrock of an esports entertainment ecosystem that extends beyond athletes to "strategists, content creators, entrepreneurs and organizers" (Steinkuehler, 2020 , p. 4). Simultaneously, its use is unbridled, leading to scholarly interventions and critical reflection on many fronts, such as diversity and inclusion, that are integral to universities (e.g., Brewer et al., 2020).

As such, Twitch is an essential site for demonstrating the interdependence and independence of gaming communities and universities. How students navigate the platform and utilize it for career building and professional development warrants investigation, along with how colleges employ Twitch for promotion, sponsorship, and communication. Our research therefore asks:

1. How does the collegiate esports community use Twitch?

2. How do players use the platform to foster independence from or interdependence with esports institutions (e.g., game publishers, administration, and Twitch itself)?

3. How do administrations and program directors use Twitch for engaging players, fans, and the university community?

\section{Methods}

As part of a larger project on collegiate esports, we are conducting in-depth, semistructured interviews with players, program directors, administrators, and students associated with esports through media outlets, gaming venues, or initiatives like graphic design. We currently have completed 19 interviews (60-120 minutes long) with contributors from five different locations. Participants were recruited via snowball sampling, starting with members of one collegiate esports program at a large US public university, then asking each interviewee to direct us to other potential sources. Interviews took place online, were transcribed and cleaned for clarity, then moved to the qualitative analysis program Dedoose. One research team member coded each interview using a grounded theory approach (e.g., generating themes from patterns in the data rather than existing hypotheses; Glaser \& Strauss, 1999). To ensure consistency between coders, the team maintained a separate shared codebook and discussed any uncertainties to determine the appropriate coding. Therefore interrater reliability scores cannot be calculated for this analysis, but are not needed due to the research's exploratory, qualitative nature; semi-structured interviews and grounded analysis lend themselves to in-depth exploration of specific phenomena rather than generalizable claims about a whole group.

\section{Preliminary Findings and Conclusion}

This study is ongoing, and we are extending our interviews in breadth (e.g., colleges and universities) and depth (e.g., players, administrators, coaches, and directors). Early analysis suggests that Twitch is important to all collegiate stakeholders, who concede it 
has a "foothold on the industry." Further, they see Twitch aspirationally, stressing its potential in the growth of collegiate esports.

Administrators' and players' perceptions of how Twitch should be utilized diverged despite these commonalities. Directors saw the service as a way to promote teams and drum up support. Getting fans to watch streams was a point of pride and an indispensable venue for sponsorship. Players aspired to be like their favorite streamers; many hoped to hit it big through streaming, or at least toyed with the idea of casting as a career. They recognized that success on Twitch required significant labor, both in terms of technical knowledge and acquiring "gamer capital" (Consalvo, 2009), citing how the best collegiate casters knew the ins and outs of the game by heart. The platform also validated their play; athletes boasted about their viewership numbers or how they taught grandparents to watch. Generally, the platform developed community connections for undergraduate players. This social aspect also had negative consequences, as athletes mentioned trolling and other toxic behavior associated with competitive gaming during their live play.

At the same time, grey areas colored the relationship between Twitch and the university. Monetization and use of streams were not clearly articulated; some directors took harsh stances on how to use Twitch, while others thought of it more as a way to let loose and have fun. Professionalism on the platform was also murky. More than one director mentioned that streamers needed to be aware that their conduct on the platform "represented" the university but did not state clear policies for use.

Our ongoing interviews will expand on these findings to investigate how Twitch is incorporated into college culture. Institutional actors seem to accept that the platform is necessary for esports' success, but have neither the knowledge nor the flexibility to adapt it into the university setting. Thus, this study will model the role specific digital platforms play in fostering independent and interdependent career paths for students, and how existing institutions may navigate those platforms as they become essential to online and offline communities.

\section{References}

Anderson, C. G., Tsaasan, A. M., Reitman, J., Lee, J. S., Wu, M., Steel, H., Turner, T., \& Steinkuehler, C. (2018). Understanding Esports as a STEM Career Ready Curriculum in the Wild. 2018 10th International Conference on Virtual Worlds and Games for Serious Applications (VS-Games), 1-6.

Baker, T. A., III, \& Holden, J. T. (2018). College eSports: A model for NCAA reform. SCL Rev., 70, 55.

Brewer, J., Romine, M., \& Taylor, T. L. (2020). Inclusion at Scale: Deploying a Community-Driven Moderation Intervention on Twitch. Proceedings of the 2020 ACM Designing Interactive Systems Conference, 757-769.

Consalvo, M. (2009). Cheating: Gaining Advantage in Videogames. MIT Press. 
Gerber, H. R. (2017). eSports and Streaming: Twitch Literacies. In Journal of Adolescent \& Adult Literacy (Vol. 61, Issue 3, pp. 343-345).

https://doi.org/10.1002/jaal.692

Glaser, B., \& Strauss, A. (1999). The Discovery of Grounded Theory: Strategies for Qualitative Research. Aldine Transaction.

Li, L., Uttarapong, J., Freeman, G., \& Wohn, D. Y. (2020). Spontaneous, Yet Studious: Esports Commentators' Live Performance and Self-Presentation Practices. Proc. ACM Hum.-Comput. Interact., 4(CSCW2), 1-25.

Richard, G. T., McKinley, Z. A., \& Ashley, R. W. (2018). Collegiate eSports as Learning Ecologies: Investigating Collaborative Learning and Cognition During Competitions. DiGRA Conference. http://www.digra.org/wp-content/uploads/digitallibrary/DIGRA_2018_paper_308.pdf

Steinkuehler, C. (2020). Esports Research: Critical, Empirical, and Historical Studies of Competitive Videogame Play. Games and Culture, 15(1), 3-8.

Taylor, N. (2020). The Numbers Game: Collegiate Esports and the Instrumentation of Movement Performance. In J. J. Sterling \& M. G. McDonald (Eds.), Sports, Society, and Technology: Bodies, Practices, and Knowledge Production (pp. 121-144). Springer Singapore. 